\title{
Electronic Communications with Patients: Improved Safety, Improved Access, or Electronic Leash-Principles and Prospects
}

\author{
Anna B. Reisman • David L. Stevens • \\ Mack Lipkin
}

Published online: 5 February 2010

(C) Springer Science+Business Media, LLC 2010

Wong and colleagues [1] suggest that Maimonides implies physicians should be available to their patients "... at all times," via cell phones. That could occasion an editorial of its own about the divine omnipresence called for, the reality that we are not available even to ourselves at all times, and that such dependence seems both impossible and damaging to a patient's sense of self-efficacy and independence. Their rhetorical device is used to introduce a discussion of cell phones, a recent but certainly not the last medium for doctor-patient communication. The authors touch upon how the core principles of communication are modified by telephonic communication and further so by cell phone use; their comments also pertain to the role of other electronic communications like e-mail, pagers,

\footnotetext{
A. B. Reisman

Department of Medicine, Yale University School of Medicine, New Haven, CT, USA

e-mail: Anna.reisman@va.gov
}

\author{
A. B. Reisman \\ Home-Based Primary Care, VA Connecticut Healthcare System, \\ 950 Campbell Ave., West Haven, CT 06516, USA \\ D. L. Stevens \\ Department of Medicine, New York University School \\ of Medicine, New York, NY, USA \\ e-mail: dls3@nyu.edu \\ D. L. Stevens \\ Department of Medicine, Gouverneur Healthcare Services, \\ 227 Madison Street, New York, NY 11215, USA \\ M. Lipkin ( $\square)$ \\ Department of Medicine, Primary Care, Primary Care Residency \\ Education Program, NYU School of Medicine, 550 First \\ Avenue, New York, NY10016, USA \\ e-mail: Mack.Lipkin@nyumc.org
}

answering services, intercoms, and devices like electrophysiologic monitors.

We propose here to add to the white noise a miasmic semblance of shape that derives from the robust literature about communication in general [2] and telephonic communication in particular [3]. When we are in an age in which many can be reached at most times, when pictures soon will be routinely added to sound, when physiological data will soon be flowing faster than drone videos from Pakistan, we need to think about how to digest all this electronic chyme. Interactive Web sites, electronic social networking, and texting have so altered the way electronically savvy individuals interact that the cell phone call may soon seem as dated as pagers. However, because doctor-patient communication lags in its adoption of new technologies-many doctors do not feel confident assessing patient needs over the phone-we will briefly review the literature on communication skills and its application to telephonic interactions.

Much writing about any sort of communications takes the form and stance of the present article: anecdotal, thoughtful but not theoretical, and free of robust scientific evidence about what actually happens, what its effects are, and how it matters to care in an empirical sense. We actually know much worth mastering about doctor-patient communication. There is an emerging consensus model based on over 10,000 articles [4], most relevant to electronic communications and cell phone use, albeit by extrapolation. In brief, whether face-to-face, on the colonoscopy table (before the injection), or on the phone, communications are about gathering information, communicating information, or developing or maintaining a relationship, which includes managing patients' feelings of dependence, independence (sometimes called activation), a sense of safety, and a sense of autonomy. Skilled 
information gathering leads to more complete and accurate data to use in clinical reasoning, greater efficiency in the (phone) encounter, and enhanced satisfaction in the care for both parties. Skilled information communication (patient education) leads to greater trust, therapeutic adherence, appointment keeping, medication taking, and satisfaction. Enhanced relationships produce improved information gathering, patient ability to comply and to change life style and behavior, healing, satisfaction, and a less anxious patient sitting alone on the other end of the phone call. Overall, better communications boils down to 60 or so behaviorally defined specific competencies. Done better, these lead to more accurate diagnoses, outcomes of care, efficiency, cost-effectiveness, and satisfaction.

By and large, the same communications principles and best practices pertain to the phone. Except that you can't see the patient, or (s)he you, so nonverbal communications-expressions, mannerisms and gestures, smells, pheromones, and aural transactions, are lost—although a few might be transiently heightened similarly but to a lesser degree as a blind person's other senses are. This blindness (soon to be partially remedied by phone/video) is the cost of the big plus: not having to be near one another to communicate. The authors focus appropriately on this big advantage of the cell phone: access, wherever (there is a signal) and whenever (the phone is on, working, and answered). The other side is also true: phone contact impedes the physician's ability to observe the patient's degree of suffering and understanding of the plan. Likewise, the patient is less likely to feel reassured by the doctor's calm demeanor or to feel safe disclosing embarrassing historical information. And poor or noisy signals, heavy accents, background noise, and concomitant sounds like breathing can make communication difficult and can mask failure to understand.

We all live with the invasive quality of cell phones that ring at the peak moment of the movie, on rounds, during our nap. There are other issues about phone use. It can be time consuming, it is not reimbursed, and it costs money to use. Voice mail can create an expectation that the message has been received and so ongoing responsibility becomes ambiguous, and, if the message hasn't been received, possibly dangerous. Delays can occur. Frustration can mount. Intrusions can pile up. This is why doctors often prefer an answering service to a pager: someone can triage, ascertain whether waiting is ok, or tell the patient to call 911. Similarly, many doctors prefer the asynchronous nature of e-mail, where no voices delude the patient into thinking the doctor has heard; and where one can elect when to engage with the flow of data and demand.

Wong et al. ask whether we should give the patient access-by providing our cell phone (or home) number. Patients love getting it-they feel respected, safe, trusted with a gift. Doctors worry about intrusion, although most report that it is seldom abused, and that is our experience. With caller ID, one can always choose not to answer. Sometimes one can monitor the message to learn if it is emergent or not. The reason most physicians give out private numbers combines caring and self-interest: better I triage this person whom I know than have them land in the ER, hospital, or morgue. Knowing the person and the case, I can make fast, simple adjustments that a stranger on call might need $20 \mathrm{~min}$ or hours to sort through and even then mess up. Consider the patient dying at home or the person with difficulty reporting symptoms who is urgently illwho wouldn't want to be available to them whenever possible? And such infrequent private phone encounters feel right - we want to be there for our patients when their need is greatest. Of course, improved phone tree systems, answering services, signout to colleagues, and the availability of physician extenders (nurse practitioners and physician's assistants) can each to some extent mitigate the need for such calls.

Wong et al. assert that doctors resent when patients call often. Their anecdote ends happily when a needy, frequent caller learns to be self-reliant and makes fewer calls. Most patients receiving a serious diagnosis will go through a similar transition from dependence and passivity to independence and self-efficacy. While we imagine that the guidance we give during the office visit will suffice for the patient to assume day-to-day management of his illness, most patients will need or benefit from additional coaching. The phone call, especially the needy one, can be seen as a "sign" of the patient not yet achieving self-reliance.

At present, we recommend common sense concerning patient boundaries as represented by the cell phone. Most patients can and will respect a private number and use it only when the need is great. The advantages of doing this are creating a sense of mutual trust (correct interdependence) and the greater efficiency and effectiveness resulting from having someone who knows the patient managing him or her when there is a problem. It is prudent not to give your private numbers to patients who have boundary issues, are obviously intrusive or not self-aware, or who are dangerous. The somatizers described by Wong et al. are not clearly exceptions to any of this. Some are dependent, many are histrionic, a few are aggressive, but most can benefit from increased phone access if handled correctly [5]. We end up giving private numbers to about $20 \%$ of our patients: especially those who will need it and those who will benefit from having the call receiver really know them and their case.

The article makes clear the need for further study and conversation about guidelines regarding access to physicians through cell phones. If you have thoughts about this, give us a call. If it is at night, call Dr. Wong. 


\section{References}

1. Wong RKM, Tan JSM, Drossman DA. Here's my phone number, don't call me: physician accessibility in the cell phone and e-mail era. Dig Dis Sci. doi:10.1007/s10620-009-1089-5.

2. Lipkin M, Putnam SM, Lazare A, eds. The Medical Interview: Clinical Care, Education, and Research. New York: Springer; 1995:643.

3. Reisman AB, Stevens D, eds. Telephone Medicine: A Guide for the Practicing Physician. Philadelphia, American College of Physicians-American Society of Internal Medicine, 2002.
4. Kalet A, Pugnaire MP, Lipkin M, et al. Teaching communications in clinical clerkships: models from the Macy Initiative in Health Communications. Acad Med. 2004;79:511-520.

5. Smith RC, Lyles JS, Gardiner JC, et al. Primary care clinicians treat patients with medically unexplained symptoms: a randomized controlled trial. J Gen Intern Med. 2006;21:671. 